\title{
Paradoxical aortic stenosis: much more than a classification problem
}

\begin{abstract}
Summary
Aortic stenosis (AS) is the most frequent valvular pathology and is one of the most common indications of valve replacement surgery. Its main form of presentation is calcified AS, whose prevalence increases proportionally with age. The indication of aortic valve replacement (AVR) is clear when the echocardiographic parameters of severity are coincident with each other and the patient presents symptoms, deterioration of the left ventricular ejection fraction (LVEF) or other indication of cardiac surgery. But with the advancement of diagnostic methods, new classifications of this condition have emerged. The term "paradoxical low-flow, low-gradient aortic stenosis" appears, which refers to the presence of an aortic valve area (AVA) $<1 \mathrm{~cm}^{2}$ or indexed AVA $<0.6 \mathrm{~cm}^{2} / \mathrm{m}^{2}$, associated with low transvalvular flow and gradients, but with preserved LVEF $(\geq 50 \%)$. This combination of data leads to a discordant classification (AVA in range of severity, medium gradient with values of mild to moderate disease). Due to the probability of potential errors that could underestimate the AVA by Doppler echo, and the uncertainty about the real meaning of this pathology, it is necessary to confirm the veracity of these findings, to give a correct diagnosis and treatment to patients with this intriguing entity.
\end{abstract}

Volume II Issue 3 - 2018

\author{
Nazar FJ \\ Hospital Juan A, Fernández, Buenos Aires, Argentina \\ Correspondence: Nazar FJ, Hospital Juan A, Fern $\tilde{A}_{j}$ dez, \\ Araoz 2873 Capital Federal, Buenos Aires, Argentina, Tel 54- \\ II33159305,Email franconazar@gmail.com
}

Received: March 19, 2018 | Published: May 292018

Keywords: Aortic valve stenosis, echocardiography, low flow

\section{Introduction}

Aortic stenosis (AS) is the valvular pathology most frequently encountered in clinical practice and is one of the most common indications of valve replacement surgery. ${ }^{1,2}$ Its main form of presentation is calcified AS, whose prevalence increases proportionally with age, affecting $2-7 \%$ of people over 65 years of age. ${ }^{3,4}$ The indication of AVR is clear when the echocardiographic parameters of severity are coincident with each other and the patient has symptoms, deterioration of the left ventricular ejection fraction (LVEF) or other indication of cardiac surgery (class I, level of evidence B), ${ }^{5,6}$ But with the advancement of technology and, consequently, of diagnostic methods, new classifications of this condition have emerged. The term "paradoxical low-flow, lowgradient aortic stenosis" (PLFLG AS) appears, which refers to the presence of an aortic valve area (AVA) $<1 \mathrm{~cm} 2$ (or indexed AVA (AVAi) $<0.6 \mathrm{~cm}^{2} / \mathrm{m}^{2}$ ) associated with low transvalvular flow and gradients, but with preserved LVEF ( $\geq 50 \%$ ). (7) This combination of data, which can be found in up to $35 \%$ of patients with severe AS and preserved LVEF, (7) leads to a discordant classification (AVA in range of severity, medium gradient with values of mild to moderate) ${ }^{5,6,8}$ The situation becomes even more complex when this subgroup of patients presents symptoms and, in general, there is a tendency to adduce potential errors that could underestimate the AVA by Doppler echo and conservative behavior is chosen, ruling out the AVR as a therapeutic option. ${ }^{9}$ For the foregoing, the need to confirm the truth of this finding is first imposed. If so, does this entity require to be considered a conventional severe AS? The objective of this review will be to try to answer these questions, after analyzing the various aspects of this intriguing entity.

\section{A new concept is born}

The entity known as low-flow and low gradient "paradoxical" aortic stenosis despite a conserved LVEF (PLFLG AS) was first described in 2007 by Hachicha et al., ${ }^{7}$ who before the clinical observation of the discordance between the echocardiographic values in these patients, they conducted a retrospective study to document the prevalence, pathophysiology and clinical relevance of this phenomenon. ${ }^{7}$ They collected data on 512 consecutive patients, both symptomatic and asymptomatic, with a mean age of $70 \pm 14$ years (range from 22 to 100 years), with severe aortic stenosis (AVA indexed $\left.\leq 0.6 \mathrm{~cm}^{2} / \mathrm{m}^{2}\right)$ and preserved $\operatorname{LVEF}(\geq 50 \%)$. Of these patients, $331(65 \%)$ had a normal stroke volume index (SVI) $\left(>35 \mathrm{ml} / \mathrm{m}^{2}\right.$, normal flow group (NF) (SVI: 36-68 ml/m²)), and $181(35 \%)$ had low flow (SVI $\leq 35 \mathrm{ml} / \mathrm{m}^{2}$, paradoxical low flow group (PLF) (SVI: 14-35 $\left.\mathrm{ml} / \mathrm{m}^{2}\right)$ ). This last group, compared with the first group, had a higher prevalence of females $(\mathrm{p}<0.05)$, lower mean transvalvular gradient $(p<0.001)$, lower LV diastolic volume index $(p<0.001)$, lower LVEF $(p<0.001)$, a higher level of global afterload, reflected in greater valvuloarterial impedance $(\mathrm{Zva})(\mathrm{p}<0.001)$ and greater age $(\mathrm{p}=0.004)$. Both groups presented a similar prevalence of risk factors and concomitant diseases. The follow-up time was $25 \pm 19$ months and the primary end point was overall mortality, regardless of whether there was AVR or not. The PLF group showed a lower overall survival at 3 years $(76 \pm 4 \%$ vs. $86 \pm 3 \%, p=0.006$, adjusted for age and gender: $\mathrm{p}=0.045$ ); in addition, within this subgroup, those treated medically had a significantly lower survival compared to those who underwent AVR (at 3 years, $58 \pm 8 \%$ vs. $93 \pm 3 \%, p=0.001$, value adjusted for age and gender: $\mathrm{p}=0.002$ ). There were no significant differences (after adjusting for sex and age) in overall survival between the NF and PLF groups when they were medically treated $(\mathrm{p}=0.15)$. A total of $65 \%$ of the patients in the NF group and $47 \%$ in the PLF group underwent AVR. In the multivariate analysis, only age (hazard ratio (HR), 1.04, $95 \%$ CI, 1.01 to $1.08, \mathrm{p}=0.025$ ), $\mathrm{Zva}>5.5 \mathrm{mmHg} / \mathrm{ml} / \mathrm{m}^{2}$ (HR, 2.6, $95 \% \mathrm{CI}, 1.2$ to $5.7, \mathrm{p}=0.017)$ and medical treatment $(\mathrm{HR}, 3.3,95 \%$ CI, 1.8 to $6.7, p=0.0003$ ) they were associated independently with a higher mortality. Thus, the AVR showed a highly protective effect. ${ }^{7}$

In this way, Hachicha et al. ${ }^{7}$ delineated the characteristics of this subgroup previously interpreted as echocardiographic inconsistencies 
and emphasized the underdiagnosis and subsequent underestimation of symptoms in these patients, with less referral to AVR and the resulting increase in mortality under medical treatment. They come to the conclusion that this pattern is consistent with a more advanced stage of the disease and has a worse prognosis. ${ }^{7}$ Although one of the questions that this new entity has received is the hypothesis of probable measurement error in the left ventricular outflow tract (LVOT); ${ }^{10}$ later, invasive hemodynamic studies confirmed the existence of this subgroup. ${ }^{11}$

\section{Definition and diagnostic difficulties}

A paradoxical low flow aortic stenosis is defined as that presented with a AVA $<1 \mathrm{~cm}^{2}$ and a stroke volume index (SVI) $<35 \mathrm{ml} / \mathrm{m}^{2}$, in the presence of a preserved LVEF ( $\geq 50 \%)$. (5-7) Due to the low flow status, in these patients generally low transvalvular gradients $(<40 \mathrm{mmHg})$ are observed despite the presence of severe valvular stenosis and preserved LVEF, hence the term "paradoxical". ${ }^{7,9,12}$ It is precisely this discordant classification that leads to uncertainty about the indication of AVR in this subgroup or, even worse, to the underestimation of the severity of the stenosis. ${ }^{7,9,12}$ In addition, the lack of correlation between the classification criteria may be related to several factors, such as small patient body surface or errors in LVOT or velocity/gradient measurement, ${ }^{12,13}$ per what these possibilities must be excluded before making the diagnosis of paradoxical AS. ${ }^{6,14}$ It is also important to bear in mind that the presence of arterial hypertension at the time of the study can lead to an erroneous diagnosis, since it could decrease the transvalvular gradients, ${ }^{14,15}$ so the severity of the disease should be re-evaluated when the patient reaches normal blood pressure values. ${ }^{6-9,16}$ Transthoracic echocardiography tends to underestimate the diameter of the LVOT partly due to its elliptical rather than circular anatomy ${ }^{17-19}$ since the smaller diameter of the elliptical section is usually measured. ${ }^{20}$ Using $3 \mathrm{D}$ transesophageal echocardiography (3D TEE), it is evident that the underestimation of the LVOT area can be close to $20 \%$ in patients with AS, which leads to a significant underestimation of the AVA. ${ }^{21,22}$ Similar data have been reported using cardiac magnetic resonance (CMR) or multislice computed tomography (MSCT) (Figure 1). ${ }^{23,24}$ Because of the above, we are faced with an entity that can be underestimated by the presence of low gradients, but that can also be overestimated, since in the presence of low flow an aortic valve with moderate disease can present a AVA in range of severity. ${ }^{25}$ The great difficulty of diagnosing this pathology is reflected in the diversity of complementary methods and the different approaches adopted by the working groups that address this issue. (26-29) In fact, because these patients usually have advanced age and multiple comorbidities, even confirming the hemodynamic data, their evaluation remains complex..$^{6,14}$
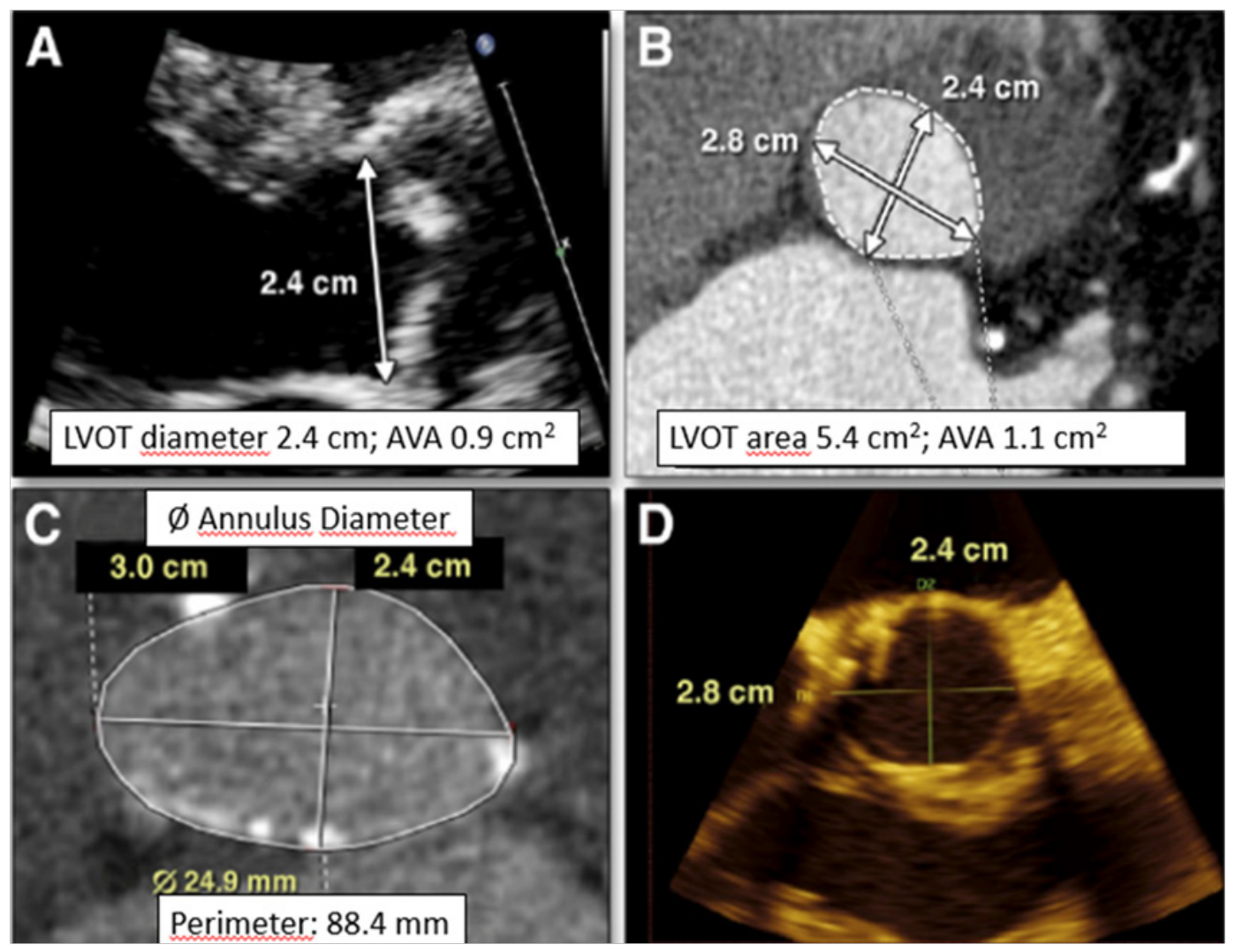

Figure I Example of underestimation of the diameter of the left ventricular outflow tract (LVOT) using 2D echocardiography in a patient with aortic stenosis. ${ }^{2}$

(A) Parasternal long axis showing a diameter of the LVOT measured at the base of the insertion of the leaflets of $2.4 \mathrm{~cm}$, resulting in a calculated aortic valve area (AVA) of $0.9 \mathrm{~cm} 2$, consistent with severe aortic stenosis (AS); (B) Computed tomography (CT) in the same patient shows a non-circular LVOT, with the same diameter of 2.4 on the minor axis plane. Using the real area of the LVOT by planimetry, the calculated AVA is I.I cm2; (C) CT scan from a different patient at the level of the aortic annulus. The annulus is elliptical with minor and major axis diameters of $2.4 \mathrm{~cm}$ and $3.0 \mathrm{~cm}$, respectively; (D) Three-dimensional transesophageal echocardiography images at the aortic annulus in the same patient also show the elliptical geometry with minor and major axis diameters of $2.4 \mathrm{~cm}$ and $2.8 \mathrm{~cm}$, very similar to computed tomography. 


\section{Characteristics of the subgroup}

\section{Epidemiology and clinical characteristics}

The prevalence of PLF AS reported in the literature is very variable and ranges between 3 and 30\% approximately (Figure 2)..$^{7,12,30-32}$ This entity is more frequent in elderly patients, predominantly in females, probably due to the fact that patients of this age group are more prone to develop LV concentric remodeling (Figure 3$).^{7}$ In addition, they most often present comorbidities such as hypertension, atrial fibrillation (AF) or diabetes mellitus (DM) compared with patients with severe aortic stenosis of high gradient (AS HG);,33 and more frequently exhibit concomitant coronary artery disease (CAD), so they are less likely to be referred for AVR. 3,4

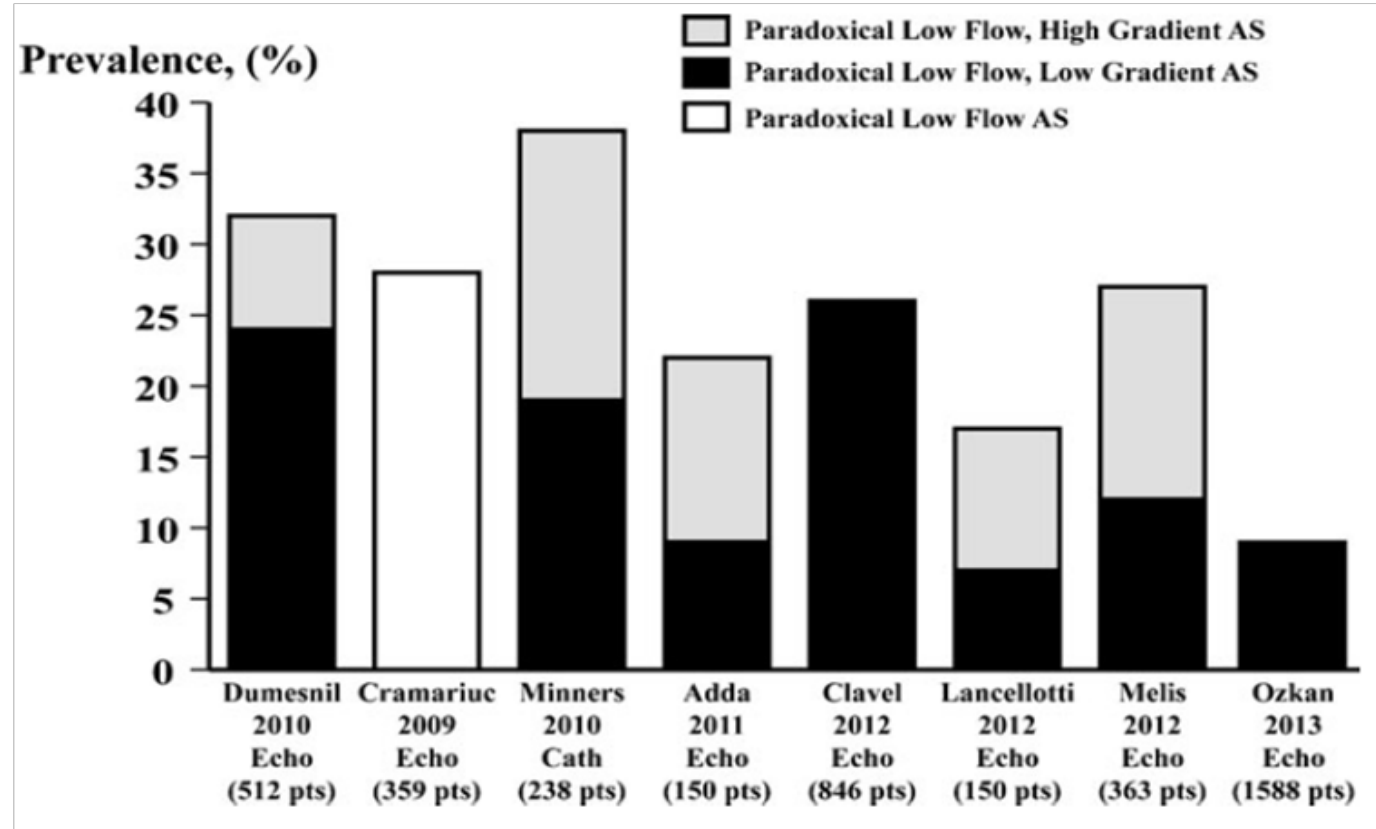

Figure 2 Prevalence of paradoxical low-flow AS, with low gradient or high gradient. ${ }^{12}$

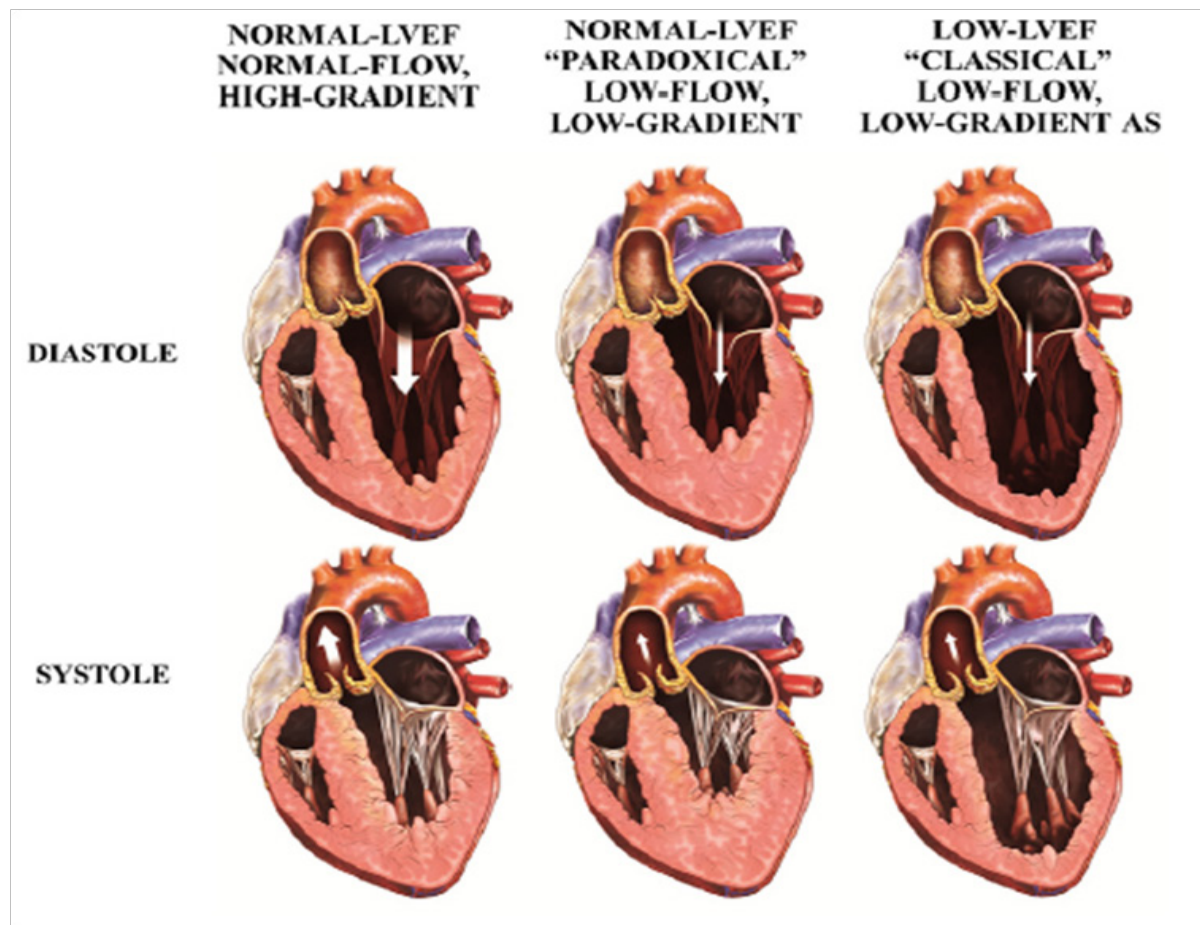

Figure 3 Different patterns of severe aortic stenosis (AS) according to flow, gradient and left ventricular geometry. ${ }^{39}$

LVEF, left ventricular ejection fraction. 


\section{Echocardiographic characteristics}

These patients typically show a pronounced concentric remodeling of the left ventricle and consequently a small cavity of the same, deterioration of the LV filling, increase of the arterial afterload and reduction of the LV longitudinal shortening..$^{7,9,12,32,35-37}$ Although they present LVEF within the normal range, previous studies show that this parameter underestimates the degree of myocardial systolic dysfunction in the presence of concentric remodeling. ${ }^{32,38}$ This can be evidenced by more sensitive echocardiographic parameters, such as the midwall fractional shortening or the global longitudinal deformation, which in these cases show significant alteration of systolic function, ${ }^{9,27,39,40}$ in relation to advanced myocardial fibrosis predominantly in the subendocardium. ${ }^{27,39}$

It is important to clarify that although the low flow state is generally associated with a low mean gradient (the gradient is a square function of the flow), in cases of very severe stenosis, the PLF may be associated with a high mean gradient despite the low SVI. This is why some authors maintain that the PLF AS entity includes patients with a low gradient (medium gradient $<40 \mathrm{mmHg}$ ) (PLF LG) and others with a high gradient (PLF HG). ${ }^{9,12}$

\section{Complementary methods}

\section{Echocardiography}

\section{Classic parameters}

The echocardiogram is, without a doubt, the most useful tool when establishing the severity of valvular heart disease, which is why the ESC/EACTS guidelines (European Society of Cardiology/European Association for Cardio-Thoracic Surgery) and ACC/AHA (American College of Cardiology/American Heart Association), among others, consider it the method of choice for that purpose; $; 5,6,20,41$ which in turn is crucial to define the surgical time. ${ }^{42}$ With the use of Doppler it is possible to know the velocities at the level of the left ventricular outflow tract (LVOT), and in this way we can apply the continuity equation to estimate the AVA. ${ }^{42}$ This parameter, together with the velocity and the transvalvular gradients (peak and medium), constitute the basis of the severity stratification of the AS. ${ }^{5}$ However, in certain occasions in which the values of these parameters are discordant among themselves, there are other variables to be taken into account, since they influence those previously mentioned.

\section{Other parameters}

At the pre-valvular level, the decrease in ventricular function translates into low transvalvular gradients. ${ }^{42}$ Although in patients with PLF AS the LVEF by definition is in normal ranges, this does not exclude intrinsic myocardial dysfunction. ${ }^{7}$ Unlike the midwall fractional shortening, LVEF is influenced both by intrinsic myocardial function and by the geometry of the LV cavity. ${ }^{7}$ This is why, for a similar degree of myocardial intrinsic shortening, any parameter based solely on endocardial displacement will tend to increase in relation to the degree of concentric remodeling. ${ }^{43}$ Therefore, it is common to observe in patients with AS and significant concentric remodeling, normal or even hypernormal LVEF, but may have incipient myocardial dysfunction (evidenced by lower midwall fractional shortening), which may contribute to low flow. ${ }^{7,35}$ Finally, the analysis of myocardial deformation (strain - strain rate) may reveal subtle alterations in myocardial contractility, even in the presence of normal LVEF. $^{32,41,44,45}$ At the valvular level, in addition to the AVA, the type of condition (etiology, type of restriction, level of calcification or degree of fibrosis) can also have a role as a determinant of the gradients and the calculations derived from them. ${ }^{42}$ The energy loss index (ELI) is a parameter that takes into account the recovery of pressure at the level of the ascending aorta and, therefore, provides a more reliable valvular gradient, avoiding the overestimation that could arise with the continuity equation. ${ }^{9,46,47}$ Another useful tool is the ratio of LVOT/ Aorta velocity, which evaluates the degree of stenosis independently of the flow. This parameter (Doppler Velocity Index) indicates how many times the blood is accelerated when passing through the aortic valve. A value of 0.25 is considered to correspond to severe AS, and implies that the blood was accelerated 4 times due to AS. ${ }^{8,9}$

With respect to the post-valvular factors, it has been established that they play a fundamental role in the PLF AS. ${ }^{7}$ At this point, the conjugation of the echocardiographic data with the taking of blood pressure during the obtaining of them, yields calculated parameters of the utmost importance. ${ }^{79}$ Thus, systemic arterial compliance, systemic vascular resistance and valvuloarterial impedance (Zva) can be obtained. ${ }^{48}$ This last parameter represents the valvular and arterial factors that oppose ventricular ejection (global afterload of the LV) by the absorption of the mechanical energy developed by the left ventricle. ${ }^{7,48}$ In simple words, it represents the cost in $\mathrm{mmHg}$ for each $\mathrm{ml}$ of blood per body surface expelled by the $\mathrm{LV}^{42}$ In turn, the vascular load is represented by aortic stiffness, peripheral resistance, systolic pressure and effective arterial elastance, which have a negative impact on systolic function. ${ }^{9,49}$ In fact, it has been shown that in patients with severe AS the longitudinal systolic function of the LV shows an inverse relationship with afterload. ${ }^{32,50}$ In this aspect, Migliore et al. ${ }^{9}$ found that post-valvular factors play a substantial role in the pathophysiology of PLF AS, and emphasize the importance of routinely calculating the ratio of TSVI/Ao and Zva integrals. ${ }^{7,9}$

\section{Eco stress (with exercise or dobutamine at low doses)}

Recently Clavel et al. demonstrated for the first time the usefulness of stress echocardiography (with exercise or dobutamine) to confirm the severity of stenosis in patients with PLFLG AS, thus being able to differentiate true AS from pseudo severe AS in this subgroup. ${ }^{25}$ In this work, they included 55 patients with severe PLFLG AS recruited in the context of two prospective observational trials, TOPAS (True Or Pseudo-Severe Aortic Stenosis) and EXERSA (Exercise Stress Echocardiography in Aortic Stenosis), ${ }^{51,52}$ the projected aortic valve area was used at a normal flow rate (AVAproj) (Figure 4) in order to evaluate the severity of the stenosis. ${ }^{25}$ This parameter was initially validated in the context of low-flow aortic stenosis and "classical" low gradient. ${ }^{51,53}$ Because all stenosis parameters are flow dependent and, in turn, the flow response to stress varies widely from one subject to another, the projected AVA allows the AVA results to be standardized according to the flow. A cut-off value $\leq 1 \mathrm{~cm}^{2}$ for the AVAproj showed a sensitivity of $92 \%$, a specificity of $100 \%$ and a correct classification percentage of $94 \%$ for the diagnosis of true PLFLG AS. ${ }^{25}$ This concept is reinforced by the fact that in those study patients who underwent AVR in the three months after the stress echocardiogram, the AVAproj showed a better correlation with the weight of the valve than any other echocardiographic parameter of rest or stress. ${ }^{25}$ While these results are promising and there were no adverse events during stress echocardiography, the small number of patients included $(n=55)$ in this study represents a limitation for its routine application in this subgroup; therefore, the viability and safety of this complementary method will have to be evaluated in a wider range of patients with PLFLG AS. ${ }^{25}$ 


$$
A V A_{\text {proj }}=\frac{A V A_{\text {peak }}-A V A_{\text {rest }}}{Q_{\text {peak }}-Q_{\text {rest }}} \times\left(250-Q_{\text {rest }}\right)+A V A_{\text {rest }}
$$

Figure 4 Formula used to calculate the projected AVA. ${ }^{25}$

AVA, aortic valve area; $\mathrm{Q}$, transvalvular flow; Peak, peak of stress; Basal, at rest.

\section{$3 D$ transesophageal echo (3D TEE)}

One of the main difficulties in the echocardiographic assessment of AS is the measurement of LVOT; and the 2D transthoracic echo (2D TTE), assuming a circular morphology of it, could underestimate the flow calculation. In contrast, 3D transesophageal echo (3D TEE) is methodologically better than 2D to measure LVOT, so it is attractive to use this method for an adequate classification of this valvular disease. While this method yields promising data, more information is required for its routine application. ${ }^{2,21}$

Cardiac magnetic resonance (CMR) and multislice computed tomography (MSCT)

The CMR can be a complementary method of use in the correct classification of AS and even to estimate the improvement of LV function after AVR. In a recent study, Barone-Rochette et al. ${ }^{54}$ analyzed a total of 128 patients ( $73 \pm 11$ years of age, 75 men) with AVAi $<0.6 \mathrm{~cm}^{2} / \mathrm{m}^{2}$ and $\mathrm{LVEF}>50 \%$ by echocardiography; who underwent CMR to measure AVA by planimetry, SVI, LV mass and focal fibrosis. They were divided by classic criteria into: normal flow/ high gradient (NFHG, $\mathrm{n}=69,54 \%$ ); low flow/high gradient (LFHG, $\mathrm{n}=28,22 \%$ ); normal flow/low gradient (NFLG, $\mathrm{n}=17,13 \%$ ) and low flow/low gradient (LFLG, $\mathrm{n}=14,11 \%$ ). The area of the LVOT, the SVI and the AVA showed good correlation between echocardiography and CMR ( $r=0.7,0.61$ and 0.65 , respectively, $\mathrm{p}<0.001$ for all). However, the AVA by planimetry (CMR) was higher in the groups with LG than in the LFHG group, and the LV mass index was lower in the two low-gradient groups than in the LFHG subgroup. All AS groups had similar LV volumes, predominant concentric remodeling/hypertrophy, and similar amounts of focal fibrosis. ${ }^{54}$

The CMR confirmed the overall accuracy of the echocardiographic classification of AS, but demonstrated that AS with LFLG and NFLG have higher AVA, less LV hypertrophy and similar focal fibrosis compared to LFHG AS. In this way, these authors found that half of the patients with severe PLG AS present AVAi $>0.6 \mathrm{~cm}^{2} / \mathrm{m}^{2}$; which puts into question the idea that LFLG AS is a more advanced stage of aortic valve disease. ${ }^{54}$ These data are discordant with those previously obtained by Herrmann et al., ${ }^{27}$ who demonstrated through myocardial fibrosis CMR in at least one segment in patients with severe LG AS, either with depressed or preserved LVEF; ${ }^{27}$ but in this work, the low gradient was associated with a higher degree of fibrosis, decreased LV longitudinal function and poorer clinical outcome, despite the preserved LVEF. Late enhancement with gadolinium has been observed mainly in the subendocardial layers of the basal segments; ${ }^{27}$ incidentally, longitudinal fibers are present mainly in the subendocardium and are more susceptible to the effects of increased parietal tension and ischemia under conditions of increased afterload. Therefore, the hypothesis that patients with extensive fibrosis would be less likely to show improvement in LV function after AVR is reasonable. ${ }^{2}$ On the other hand, the MSCT, in addition to offering the planimetry of the LVOT and the AVA, provides data on the valvular calcium load; which, as has recently been demonstrated, in patients with AS and discordance (LG and AVAi congruent with severity), is strongly associated with severity. In this way, it would allow differential diagnosis and help in the management of this complex subgroup (Table 1).55

\begin{tabular}{|c|c|c|c|c|c|}
\hline Type of Aortic Stenosis & $\begin{array}{c}\text { Mean } \\
\text { Gradient } \\
(\mathrm{mm} \mathrm{Hg})\end{array}$ & $\begin{array}{l}\text { LVEF } \\
\text { (\%) }\end{array}$ & $\begin{array}{l}\text { LV Stroke } \\
\text { Volume } \\
\text { Index }\left(\mathrm{ml} / \mathrm{m}^{2}\right)\end{array}$ & $\begin{array}{l}\text { Assessment } \\
\text { of Contractile } \\
\text { Reserve }\end{array}$ & $\begin{array}{c}\text { Elevated } \\
\text { Computed } \\
\text { Tomography } \\
\text { AV Calcium } \\
\text { Score }\end{array}$ \\
\hline Normal flow high gradient & $\geq 40$ & $\geq 50$ & $>35$ & - & + \\
\hline $\begin{array}{l}\text { Normal flow and } \\
\text { gradient, low EF }\end{array}$ & $\geq 40$ & $<50$ & $>35$ & - & + \\
\hline $\begin{array}{l}\text { Classical low flow, } \\
\text { low gradient }\end{array}$ & $<40$ & $<50$ & $\leq 35$ & + & + \\
\hline $\begin{array}{l}\text { Paradoxical low flow, } \\
\text { low gradient }\end{array}$ & $<40$ & $\geq 50$ & $\leq 35$ & $+1-$ & + \\
\hline Pseudoaortic stenosis & $<40$ & $<50$ & $\leq 35$ & - & - \\
\hline
\end{tabular}

Table I Subtypes of aortic stenosis and utility of complementary methods ${ }^{29}$ 


\section{Conclusion}

This entity reinforces the need for a more complete assessment of the severity of AS, which goes beyond the classical measures, to include parameters that are less dependent on flow. A more complete assessment of LV function should be performed and not be based solely on parameters that reflect displacement of the endocardium. In short, there is no isolated value, despite the great utility of the transvalvular gradient, which can predict the need for valve replacement or appearance of symptoms. Given the intense debate about the indication of AVR in patients with PLFLG AS, and even the questioning of its severity, it is evident that the only way to avoid this limitation is through the use of data from controlled, prospective and randomized clinical trials that allow determine the true severity of this pathology.

\section{Conflict of interests}

Any.

\section{References}

1. Lung B, Vahanian A. Epidemiology of valvular heart disease in the adult. Nat Rev Cardiol. 2011;8(3):162-172.

2. Tandon A, Grayburn PA. Imaging of low gradient severe aortic stenosis. J Am Col Cardiol Img. 2013;6(2):184-95.

3. Nkomo VT, Gardin JM, Skelton TN, et al. Burden of valvular heart diseases: a population-based study. Lancet. 2006;368(9540):1005-11.

4. Thaden JJ, Nkomo VT, Enriquez-Sarano M. The global burden of aortic stenosis. Prog Cardiovasc Dis. 2014;56(6):565-71.

5. Nishimura RA, Otto CM, Bonow RO, et al. 2014 AHA/ACC Guideline for the Management of Patients with Valvular Heart Disease: a report of the American College of Cardiology/American Heart Association Task Force on Practice Guidelines. J Am Coll Cardiol. 2014;129(23):2440-92.

6. Vahanian A, Alfieri O, Andreotti A, et al. Guidelines on the management of valvular heart disease (version 2012): The Joint Task Force on the Management of valvular heart disease of the European society of cardiology (ESC) the European Association for Cardio-Thoracic Surgery (EACTS). Eur Heart J. 2012;33(19):2451-96.

7. Hachicha Z, Dumesnil JG, Bogaty P, et al. Paradoxical low flow, low gradient severe aortic stenosis despite preserved ejection fraction is associated with higher afterload and reduced survival. Circulation. 2007;115(22):2856-64.

8. Lax J, Stutzbach P. Consenso de valvulopatías / versión resumida. Rev Argent Cardiol 2015;83:68-80.

9. Migliore R, Adaniya M, Mantilla D, et al. Carga vascular y valvular en la estenosis aórtica grave con bajo flujo, bajo gradiente y fracción de eyección normal. Rev Argent Cardiol. 2010;78:30-38.

10. Jander N. Low-gradient 'severe' aortic stenosis with preserved ejection fraction: new entity, or discrepant definitions? Eur Heart J. 2008;10:E1115.

11. Lauten J, Rost C, Breithardt OA, et al. Invasive hemodynamic characteristics of low gradient severe aortic stenosis despite preserved ejection fraction. J Am Coll Cardiol. 2013;61(17):1799-808.

12. Clavel MA, Pibarot P, Dumesnil JG. Paradoxical low flow aortic valve stenosis: incidence, evaluation, and clinical significance. Curr Cardiol Rep. 2014;16(1):431.
13. Michelena H, Margaryan E, Miller FA, et al. Inconsistent echocardiographic grading of aortic stenosis: is the left ventricular outflow tract important? Heart. 2013;99(13):921-31.

14. Zoghbi WA. Low gradient "severe" aortic stenosis with normal systolic function: time to refine the guidelines? Circulation. 2011;123(8):838-40.

15. Jander N, Minners J, Holme I, et al. Outcome of patients with lowgradient "severe" aortic stenosis and preserved ejection fraction. Circulation. 2011;123(8):887-95.

16. Baumgartner H, Hung J, Bermejo J, et al. Echocardiographic assessment of valve stenosis: EAE/ASE recommendations for clinical practice. Eur J Echocardiogr. 2009;10:1-25.

17. Baumgartner H, Kratzer H, Helmreich G, et al. Determination of aortic valve area by Doppler echocardiography using the continuity equation: a critical evaluation. Cardiology. 1990;77(2):101-111.

18. Doddamani S, Bello R, Friedman MA, et al. Demonstration of left ventricular outflow tract eccentricity by real time $3 \mathrm{D}$ echocardiography: implications for the determination of aortic valve area. Echocardiography. 2007;24(8):860-866.

19. Burgstahler C, Kunze M, Loffler C, et al. Assessment of left ventricular outflow tract geometry in non-stenotic and stenotic aortic valves by cardiovascular magnetic resonance. J Cardiovasc Magn Reson. 2006;8(6):825-829.

20. Evangelista A, Galian L. Estenosis aórtica grave con bajo flujo paradójico. Aclarando el misterio. Rev Argent Cardiol. 2015;83:279-281.

21. Lombardero M, Henquin R, Perea G, et al. Eco 3D transesofágico en la estenosis aórtica con bajo flujo/bajo gradiente paradójico. Rev Argent Cardiol. 2015;83:328-334.

22. Gaspar T, Adawi S, Sachner R, et al. Three-dimensional imaging of the left ventricular outflow tract: impact on aortic valve area estimation by the continuity equation. J Am Soc Echocardiogr. 2012;25(7):749-57.

23. Doddamani S, Grushko MJ, Makaryus AN, et al. Demonstration of left ventricular outflow tract eccentricity by 64 -slice multi-detector CT. Int J Cardiovasc Imaging. 2009;25(2):175-81.

24. Chin CW, Khaw HJ, Luo E, et al. Echocardiography underestimates stroke volume and aortic valve area: implications for patients with smallarea low-gradient aortic stenosis. Can J Cardiol. 2014;30(9):1064-72.

25. Clavel MA, Ennezat PV, Maréchaux S, et al. Stress echocardiography to assess stenosis severity and predict outcome in patients with paradoxical low-flow, low-gradient aortic stenosis and preserved LVEF. JACC Cardiovasc Imaging. 2013;6(2):175-83.

26. Awtry E, Davidoff R. Low-flow/low-gradient aortic stenosis. Circulation. 2011;124:739-41.

27. Herrmann S, Stork S, Niemann M, et al. Low-gradient aortic valve stenosis: Myocardial fibrosis and its influence on function and outcome. J Am Coll Cardiol. 2011;58(4):402-12.

28. Cueff C, Serfaty JM, Cimadevilla C, et al. Measurement of aortic valve calcification using multislice computed tomography: correlation with haemodynamic severity of aortic stenosis and clinical implication for patients with low ejection fraction. Heart. 2011;97(9): 721-6.

29. Anjan VY, Herrmann HC. All aortic stenoses are not created equal. J Am Coll Cardiol. 2015;65(7):654-6.

30. Lancellotti P, Magne J, Donal E, et al. Clinical outcome in asymptomatic severe aortic stenosis: insights from the new proposed aortic stenosis grading classification. J Am Coll Cardiol. 2012;59(3):235-43. 
31. Eleid MF, Sorajja P, Michelena HI, et al. Flow-gradient patterns in severe aortic stenosis with preserved ejection fraction: clinical characteristics and predictors of survival. Circulation. 2013;128(16):1781-1789.

32. Adda J, Mielot C, Giorgi T, et al. Low-flow, low-gradient severe aortic stenosis despite normal ejection fraction is associated with severe left ventricular dysfunction as assessed by speckle-tracking echocardiography: a multicenter study. Circ Cardiovasc Imaging. 2012;5(1):27-35.

33. Rosenhek R, Zilberszac R, Schemper M, et al. Natural history of very severe aortic stenosis. Circulation. 2010;121(1):151-156.

34. Maes F, Boulif J, Pierard S, et al. Natural history of paradoxical low gradient "severe" aortic stenosis. Circ Cardiovasc Img. 2014;7(4):714 22.

35. Cramariuc D, Cioffi G, Rieck AE, et al. Low-flow aortic stenosis in asymptomatic patients. J Am Coll Cardiol Img. 2009;2(4):390-9.

36. Mehrotra P, Jansen K, Flynn AW, et al. Differential left ventricular remodelling and longitudinal function distinguishes low flow from normal flow preserved ejection fraction low gradient severe aortic stenosis. Eur Heart J. 2013;34(2):1906-1914.

37. Ramírez JD, Fernández R, Muñoz E, et al. Estenosis aórtica severa con fracción de expulsión normal de flujo bajo paradójico y bajo gradiente. Rev Colomb Cardiol. 2014;21(2):76-83.

38. Dumesnil JG, Shoucri RM. Effect of the geometry of the left ventricle on the calculation of ejection fraction. Circulation. 1982;65:91-8.

39. Pibarot P, Dumesnil JG. Low-flow, low-gradient aortic stenosis with normal and depressed left ventricular ejection fraction. $J$ Am Coll Cardiol. 2012;60(19):1845-53.

40. Lee SP, Kim YJ, Kim JH, et al. Deterioration of myocardial function in paradoxical low-flow severe aortic stenosis: two-dimensional strain analysis. J Am Soc Echocardiogr. 2011;24(9):976-83.

41. Tarasoutchi F, Montera MW, Grinberg M, et al. Directriz Brasileña de Valvulopatías - SBC 2011 / I Directriz Interamericana de Valvulopatías - SIAC 2011. Arq Bras Cardiol. 2011;97(5):1-67.

42. Prado A, Hasbani E, Stoermann W. Estenosis aórtica de bajo flujo paradójico. Cuando la información del ecocardiograma no es congruente. Rev Fed Arg Cardiol. 2012;41(1):14-16.

43. Migliore RA, Chianelli O, Adaniya ME, et al. Evaluación de la función sistólica en la estenosis aórtica mediante la fracción de acortamiento mesoparietal. Su relación con la hipertrofia. Rev Argent Cardiol. 2004; $72: 439-44$

44. Delgado V, Tops LF, van Bommel RJ, et al. Strain analysis in patients with severe aortic stenosis and preserved left ventricular ejection fraction undergoing surgical valve replacement. Eur Heart J. 2009;30(24):303747.
45. Laurenzano CS. Pronóstico y tratamiento de la estenosis aórtica severa con bajo gradiente y fracción de eyección conservada. Revista Conarec. 2013;29:138-147.

46. García D, Pibarot P, Dumesnil JG, et al. Assessment of aortic valve stenosis severity: a new index based on the energy loss concept. Circulation. 2000;101(7):765-71.

47. García D, Dumesnil JG, Durand L-G, et al. Discrepancies between catheter and Doppler estimates of valve effective orifice area can be predicted from the pressure recovery phenomenon. J Am Coll Cardiol. $2003 ; 41(3): 435-42$.

48. Briand M, Dumesnil JG, Kadem L, et al. Reduced systemic arterial compliance impacts significantly LV afterload and functions in aortic stenosis: implications for diagnosis and treatment. J Am Coll Cardiol. 2005;46(2):291-298.

49. Migliore RA, Adaniya ME, Barranco M, et al. Efecto de la elastancia arterial efectiva sobre la función sistólica ventricular izquierda en la estenosis aórtica grave. Rev Argent Cardiol. 2008;76(4):278-285.

50. Migliore RA, Adaniya ME, Barranco M, et al. La función sistólica longitudinal del ventrículo izquierdo, la poscarga y la contractilidad en la estenosis aórtica grave. Rev Argent Cardiol. 2015;83:321-327.

51. Clavel MA, Burwash IG, Mundigler G, et al. Validation of conventiona and simplified methods to calculate projected valve area at norma flow rate in patients with low flow, low gradient aortic stenosis: the multicenter TOPAS (True or Pseudo Severe Aortic Stenosis) study. J Am Soc Echocardiogr. 2010;23(4):380-6.

52. Maréchaux S, Hachicha Z, Bellouin A, et al. Usefulness of exercise stress echocardiography for risk stratification of true asymptomatic patients with aortic valve stenosis. Eur Heart J. 2010;31(11):1390-7.

53. Blais C, Burwash IG, Mundigler G, et al. Projected valve area at normal flow rate improves the assessment of stenosis severity in patients with low flow, low-gradient aortic stenosis: The multicenter TOPAS (Truly or Pseudo Severe Aortic Stenosis) study. Circulation. 2006;113(5):711-21.

54. Barone-Rochette G, Piérard S, Seldrum S, et al. Aortic valve area, stroke volume, left ventricular hypertrophy, remodeling, and fibrosis in aortic stenosis assessed by cardiac magnetic resonance imaging: comparison between high and low gradient and normal and low flow aortic stenosis. Circ Cardiovasc Img. 2013;6(6):1009-17.

55. Clavel MA, Messika-Zeitoun D, Pibarot $\mathrm{P}$, et al. The complex nature of discordant severe calcified aortic valve disease grading: new insights from combined Doppler echocardiographic and computed tomographic study. J Am Coll Cardiol. 2013;62(24):2329-38. 